\title{
China vaccine scandal rattles public's confidence and tests government
}

Xin Chen ${ }^{1}$, Liang $\mathrm{Gao}^{2, *}$

${ }^{1}$ Department of Urology, The Second Affiliated Hospital of Anhui Medical University, Hefei, China

${ }^{2}$ Institute of Experimental Orthopaedics and Osteoarthritis Research, Saarland University, Homburg/Saar, Germany

${ }^{*}$ Corresponding to:

Liang Gao, MD, Institute of Experimental Orthopaedics and Osteoarthritis Research, Saarland University, Kirrberger Street 100, Building 37, Homburg/Saar, 66421, Germany liang.gao@uni-saarland.de 
Outrage swept Chinese social media in July 2018, as the China Food and Drug Administration exposed a vaccine safety scandal that two of the country's largest vaccine makers had been providing defective children vaccines. ${ }^{1} 650,000$ doses of diphtheria, pertussis, and tetanus (DPT) vaccines and 110,000 doses of rabies vaccines did not reach the potency standard and sold in at least 10 provincial areas. ${ }^{2}$

Within 48 hours after this scandal goes viral on social media, President Xi Jinping promised the government would "investigate to the end". "It is necessary to promptly release the progress of the investigation and effectively respond to the concerns of the people". ${ }^{3}$ Local police have arrested 18 executives of the companies and multiple investigations are now under way, involving multiple regulators, securities inspectors, and the anti-corruption unit.

The Chinese National Health Commission reported no cases of diphtheria, 10390 cases of pertussis (none fatal) and 93 cases of tetanus ( 3 fatal) in $2017 .{ }^{4}$ The World Health Organization recorded 516 cases (502 fatal) of rabies in China, which represents the second highest number of reported cases worldwide in last year. Noteworthily, this significantly high mortality rate $(97.3 \%)$ indicates the critical necessity for proper vaccination. ${ }^{5}$

It is still unclear how many children received those substandard vaccines and there have been no reports of injuries or side effects. To restore the public's confidence, the government need to explore the remedies for defective vaccines, improve the health regulation system, and ensure the timely communication to the public. 
We declare no competing interests.

\section{References}

1. China Food and Drug Administration.

http://cnda.cfda.gov.cn/WS04/CL2056/329593.html (accessed August 06, 2018; in Chinese).

2. Where does the problem vaccine distribute? 30 provinces responded and many have stopped using them. http://www.chinanews.com/gn/2018/07-23/8575974.shtml (accessed August 06, 2018; in Chinese).

3. Xi Jinping made important instructions on Jilin Changchun Longevity Biological Vaccine Case. http://www.xinhuanet.com/politics/2018-07/23/c_1123166080.htm (accessed August 06, 2018; in Chinese).

4. World Health Organization Western Pacific Region. Rabies.

http://www.wpro.who.int/china/mediacentre/factsheets/rabies/en/ (accessed August 06, 2018).

5. National Health and Family Planning Commission. Overview of the national legal infectious disease epidemic in 2017.

www.nhfpc.gov.cn/jkj/s3578/201802/de926bdb046749abb7b0a8e23d929104.shtml (accessed August 06, 2018; in Chinese). 